\title{
Reúso da água da despesca na produção de camarão
}

\author{
Renato C. Leitão ${ }^{1}$, Regina R. R. Cavalcante' ${ }^{2}$, Esaú M. Ribeiro ${ }^{3}$, \\ Rayanne L. Claudino ${ }^{4}$, Naylson M. Maciel ${ }^{1}$ \& Morsyleide de F. Rosa ${ }^{1}$
}

\begin{abstract}
RESUMO
A carcinicultura causa impactos ambientais significativos devidos, sobremaneira, à elevada demanda de água e ao descarte de efluentes, agravando o problema de assoreamento e poluição dos mananciais. Este trabalho teve como objetivo buscar uma alternativa para o reaproveitamento do efluente da carcinicultura na própria atividade e assim mitigar os impactos causados. Os experimentos foram realizados em três etapas, a primeira relacionada à toxicidade, para as pós-larvas das águas de despesca, a segunda com a mortalidade das pós-larvas em curto prazo e a terceira relacionada com a sobrevivência em longo prazo e produtividade do camarão ao longo de um ciclo de engorda, utilizando-se água de despesca. Os resultados mostraram que o índice de sobrevivência é de 42,1\% quando o camarão é cultivado em águas de despesca; no entanto, a maior mortalidade dos camarões ocorre principalmente na primeira semana do ciclo de engorda, indicando que uma mudança de estratégia na produção de camarão pode aumentar a produtividade da fazenda.
\end{abstract}

Palavras-chave: camarão, água residuária, reciclagem, produtividade

\section{Reuse of the shrimp farm effluents in the production of shrimp}

\begin{abstract}
The shrimp farming cause significant environmental impacts due to high water consumption and effluent discharge, which worsen problems of sediment deposition and water pollution. This study aims to find an alternative for the use of shrimp farming effluent, and to mitigate the aforementioned impacts. The experiments were carried out in three phases, where the first dealt with the toxicity of the reused effluent on the post-larvae of shrimp, the second dealt with the short term mortality of the shrimps and the third dealt with the long-term survival and productivity of the shrimps along the entire growing cycle. The results show that the survival index is $42.1 \%$ when the shrimps are cultivated in shrimp farm effluent. However, most of the mortality occurred within the first week of the growing cycle. This indicates that a change in production strategy can increase productivity of the farm.
\end{abstract}

Key words: shrimp, wastewater, recycle, productivity

Embrapa Agroindústria Tropical. Rua Dra. Sara Mesquita 2270, Planalto do Pici, CEP 60511-110 Fortaleza, CE, Fone: (85) 3391-7213, Fax: 3391-7109. E-mail: renato@cnpat.embrapa.br; morsy@cnpat.embrapa.br.

${ }^{2}$ Empresa de Assistência Técnica e Extensão Rural do Ceará. Avenida Bezerra de Menezes 1900, São Gerardo, CEP 60725-901 Fortaleza, CE, Fone: (85) 3101-7630, E-mail: regina.regia@ematerce.ce.gov.br.

${ }^{3}$ Instituto Agropolos do Ceará. Rua Barão de Aratanha 1450, CEP: 60050-071 Fortaleza, CE, Fone: (85) 3101-1670, Fax: (85) 3101-1679, E-mail: esaumribeiro@gmail.com.

${ }^{4}$ Universidade Federal do Ceará. Centro de Ciências Agrárias, Av Mister Hull, 2977, Antonio Bezerra. CEP 60021-970 Fortaleza, CE, Fone: (85) 3366-9732. E-mail: rayanneclaudino@yahoo.com.br 


\section{INTRODUÇÃO}

A carcinicultura se intensificou no Nordeste do Brasil, sobretudo no Estado do Ceará, graças à rentabilidade econômica e elevada produtividade. Entre os anos 2000 e 2003 foram instaladas 32 fazendas de camarão ao longo da região do Baixo Jaguaribe, nos municípios de Russas, Quixeré, Jaguaruana e Itaiçaba (Figueiredo et al., 2006). A espécie utilizada para o cultivo é o Litopenaeus vannamei (camarão branco do pacífico) que, de acordo com Miranda et al. (2010), apresenta ótima taxa de sobrevivência em baixa salinidade (valores entre 0,5 a $28,5 \mathrm{mg} \mathrm{L}^{-1}$ ) (Spanghero et al., 2008), boa conversão alimentar, bom crescimento e bom rendimento no cozimento; além disso, possui grande aceitação nos mercados americano, asiático, europeu e brasileiro.

Passada a expansão inicial exagerada de novas áreas de cultivo, atingiu-se um estágio de equilíbrio no qual os carcinicultores que prosperaram no empreendimento se estabeleceram no mercado. Nesta nova fase surgiram outras preocupações no setor, tais como aumento da produção com garantia de qualidade do produto, busca de mercados mais rentáveis, aparecimento de doenças, procura da ração mais adequada, qualidade da água nos viveiros, etc., assim como novas cobranças das autoridades competentes, exigindo alvará de funcionamento, relatório de impacto ambiental, caracterização dos efluentes lançados nos corpos receptores, etc.

Soares et al. (2007) utilizando imagens orbitais do satélite LANDSAT e cartas topográficas digitalizadas dos anos de 1999, 2002 e 2005, realizaram um mapeamento e levantamento das áreas ocupadas com atividades de carcinicultura no estuário do rio Jaguaribe, Estado do Ceará, e observaram aumento significativo da área ocupada com esta atividade, e estimaram que em 1999 existiam 408,69 ha de fazendas de camarão em cativeiro, em 2002 eram 909,32 ha e em 2005, $1.676,78$ ha. Segundo informações fornecidas por técnicos da Superintendência Estadual do Meio Ambiente do Ceará (SEMACE), em 2011 foram contabilizadas 55 fazendas nos municípios de Russas, Quixeré, Jaguaruana e Itaiçaba.

Figueiredo et al. (2003) apresentaram dados socioeconômicos e ambientais mostrando que a carcinicultura representava a segunda maior atividade demandante de água na região semiárida do Baixo Jaguaribe, fato que origina conflitos pela água entre os diferentes usuários dessa bacia hidrográfica (comunidades urbanas, irrigantes e carcinicultores), onde a escassez é uma constante.

De acordo com Wang (2003), para manter as taxas de crescimento da atividade em níveis sustentáveis, deve-se buscar meios para diminuir a quantidade de água demandada e de efluente produzido, sem prejudicar o grau de resistência das espécies às doenças. A racionalização do uso da água torna-se então uma ação estratégica e indispensável. Ao mesmo tempo, os efluentes dos viveiros de camarão, ricos em nutrientes e matéria orgânica, podem contribuir para mudanças no estado trófico dos corpos hídricos receptores (Castello et al., 2008; Alencar et al., 2010) portanto, precisam ser adequadamente tratados antes da descarga final. Sandu et al. (2002) afirmam que se fossem introduzidos métodos de reciclagem e reúso nas atividades produtivas, a descarga final poderia ser reduzida em até $90 \%$, o que representaria uma economia considerável de água. Sistemas de recirculação que integram processos de tratamento de efluentes têm ganhado interesse em nível mundial (Lin et al., 2003). Miranda et al. (2007), comparando a água proveniente da descarga de tanques de camarão com a água do rio Jaguaribe para irrigação de melão e arroz, observaram que não houve diferença significativa na produção de arroz quando foram utilizados $100 \%$ da adubação por N-P-K recomendada. Além disto, os autores constataram que a produção de arroz com o efluente foi superior quando se aplicou adubação com $75 \%$ da dose recomendada. As produções de melão obtidas com águas de despesca e águas do rio foram semelhantes, indicando que as atividades de produção de camarão e a agricultura irrigada podem ser combinadas para aumento da rentabilidade dos produtores e diminuição dos impactos ambientais. Uma alternativa para os efluentes da carcinicultura é o reúso em cultivo de vegetais através de hidroponia, o que aumenta a remoção de nutrientes melhorando a qualidade de água antes de seu retorno aos viveiros (Castellani et al., 2009)

O maior consumo de água na carcinicultura ocorre nas trocas de água no viveiro e na despesca. O descarte de efluentes para os corpos hídricos pode acarretar mudanças nas características da água e efeitos negativos sobre a biota, já que a água proveniente dos cultivos é rica em matéria orgânica e sólidos em suspensão. Para promover economia da água e, consequentemente, menor impacto dos efluentes sobre os ecossistemas receptores, a Resolução n. 312/02 do CONAMA propõe que devem ser construídos bacia de sedimentação ou decantação e sistema de recirculação de água (Brasil, 2002). Segundo Joventino \& Mayorga (2008), 95\% dos empreendimentos com carcinicultura da Região de Fortim, Estado do Ceará, não possuíam bacia de sedimentação e nenhum deles fazia recirculação de água na propriedade.

A duração do cultivo na fase de engorda do camarão pode durar de 3 a 8 meses. Valenti (2002) estima que a produção de camarão em água doce seja de aproximadamente $3.000 \mathrm{~kg} \mathrm{ha}^{-1}$ ano $^{-1}$, levando-se em consideração: dois cultivos de engorda de 6 meses cada um, período de berçário de 60 dias, sobrevivência final de $70 \%$ com peso médio final de $30 \mathrm{~g}$. Com a utilização da água de despesca para produção do camarão é possível aumentar a produtividade do sistema visto que existe um aproveitamento dos nutrientes (Alencar et al., 2010).

Objetivou-se com este trabalho avaliar o impacto do reúso das águas de despesca na produção do camarão Litopenaeus vannamei, através de testes de toxicidade, avaliação da sobrevivência nos viveiros, tempo do ciclo de engorda e parâmetros biométricos.

\section{Material e MÉTODOS}

As investigações sobre o reúso das águas de despesca na produção do camarão foram realizadas em três etapas, a primeira relacionada com a toxicidade, para as pós-larvas das águas de despesca; a segunda etapa se relaciona com a mortalidade das pós-larvas em curto prazo; finalmente, foram realizados 
experimentos relacionados com a sobrevivência e produtividade do camarão em longo prazo, durante um ciclo de engorda, utilizando-se água de despesca.

Os trabalhos com toxicidade in vitro foram realizados no Laboratório de Gestão Ambiental na Embrapa Agroindústria Tropical, Fortaleza, CE, e as etapas de campo, na fazenda Poço da Onça, localizada no município de Russas, região do Baixo Jaguaribe, no Estado do Ceará, latitude $4^{\circ} 58$ ' 58,03" S, longitude $37^{\circ} 54^{\prime} 34,61^{\prime \prime} \mathrm{O}$. O clima na região é semiárido, com classificação de Köppen Bsh (muito quente e seco) e a precipitação média é de $720 \mathrm{~mm}$. Aempresa possui dois viveiros de $30.000 \mathrm{~m}^{2}$ cada um e adota o sistema de cultivo de Litopenaeus vannamei por fertilização e aeração dos viveiros. As pós-larvas cultivadas são adquiridas de terceiros que executam atividade de larvicultura. Os ciclos de engorda duram entre 100 e 150 dias, a densidade populacional varia entre 35 e 50 pós-larvas $\cdot \mathrm{m}^{-2}$, produzindo entre 7000 e $12000 \mathrm{~kg}$ de camarão em cada ciclo.

\section{Etapa 1 - Teste de toxicidade in vitro}

Para que se possa implementar a recirculação de efluentes da carcinicultura em viveiros é necessário o exame da toxicidade deste efluente para o camarão, cujo estudo durou sete dias, sendo testadas 5 proporções de água do rio e água de despesca, em aquários de 2 L (Tabela 1), com 20 pós-larvas de camarão em cada aquário, com 3 repetições, totalizando 15 aquários. As pós-larvas de camarão da espécie Litopenaeus vannamei e as águas foram provenientes da fazenda Poço da Onça; os aquários foram aerados continuamente.

Tabela 1. Descrição dos tratamentos (Experimento 1)

\begin{tabular}{ccc}
\hline Tratamento & \% água rio & \% água despesca \\
T1 & 100 & 0 \\
T2 & 75 & 25 \\
T3 & 50 & 50 \\
T4 & 25 & 75 \\
T5 & 0 & 100 \\
\hline
\end{tabular}

Todos os dias esses indivíduos eram alimentados com $8 \%$ do seu peso vivo, ou seja, $0,04 \mathrm{~g}$ de ração, duas vezes por dia, e feitas a medição de $\mathrm{pH}$ e a observação da mortandade dos indivíduos. Além disso, a cada dois dias foram realizadas uma drenagem de $20 \%$ da água dos aquários e a substituição por água de despesca ou água do rio, dependendo do tratamento.

\section{Etapa 2 - Teste de toxicidade in loco}

Para estudar a toxicidade das águas de despesca em condições mais próximas das reais utilizaram-se 12 reservatórios de plástico de 1000 L cada um. Cada grupo de dois reservatórios foi preenchido com uma mistura de água da despesca e água do rio, em diferentes proporções e deixado em repouso durante 15 dias, que é o tempo normal entre uma despesca e um novo povoamento do viveiro. Cada reservatório foi povoado com 50 unidades de pós-larvas, com exceção de dois deles, que foram preenchidos com $100 \%$ de água do rio Jaguaribe, os quais foram utilizados para controle. Esta população corresponde a uma densidade de 50 unidades por metro quadrado, que é a praticada na fazenda em estudo. A Figura 1 apresenta a disposição dos reservatórios em campo; a nomenclatura utilizada de acordo com a proporção entre a água de despesca e a água do rio Jaguaribe, está apresentada na Tabela 2.
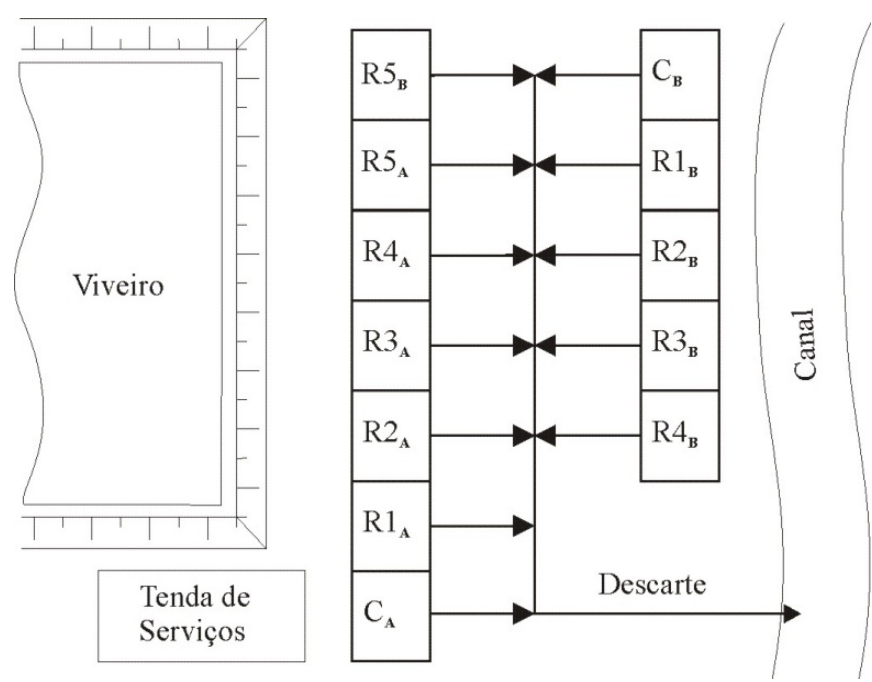

Obs.: R - Reservatório; A - Réplica 1; B - Réplica 2; C - Controle

Figura 1. Disposição dos reservatórios para teste de toxicidade in loco

Tabela 2. Nomenclatura utilizada no teste de toxicidade in loco, conforme a Figura 1

\begin{tabular}{cccc}
\hline Unidade $^{*}$ & $\begin{array}{c}\text { \% Água de } \\
\text { despesca rio }\end{array}$ & \% Água de rio & $\begin{array}{c}\text { Indivíduos } \\
\text { povoados }\end{array}$ \\
C & 0 & 100 & 0 \\
R1 & 100 & 0 & 50 \\
R2 & 75 & 25 & 50 \\
R3 & 50 & 50 & 50 \\
R4 & 25 & 75 & 50 \\
R5 & 0 & 100 & 50 \\
\hline${ }^{*}$ C - controle; R - reservatório & &
\end{tabular}

As pós-larvas foram obtidas em laboratórios do Rio Grande do Norte e transportadas para os berçários da fazenda Poço da Onça, onde foram aclimatadas durante duas semanas. Para desenvolvimento do experimento foram escolhidos, aleatoriamente, indivíduos juvenis (com comprimento entre $9 \mathrm{e}$ $13 \mathrm{~mm}$ ), por serem mais sensíveis às substâncias tóxicas presentes no meio gerando, portanto, respostas mais rápidas (Allan et al., 1990; Zhao et al., 1997; Barajas et al., 2006). Os reservatórios foram aerados constantemente mantendo-se condições ótimas para o oxigênio dissolvido (> $\left.6 \mathrm{mg} \mathrm{L}^{-1}\right)$, salinidade $( \pm 5 \%)$, pH $(7$ a 8,5$)$ e temperatura $\left(25\right.$ a $\left.29^{\circ} \mathrm{C}\right)$. As pós-larvas foram alimentadas com ração e Artemia nauplii.

Wajsbrot et al. (1990) afirmam que em qualquer ensaio de toxicidade com camarão se deve garantir duração mínima para o experimento, de $96 \mathrm{~h}$; em período menor as mudanças fisiológicas poderiam confundir os resultados do experimento e, assim sendo, convencionou-se que os testes teriam duração de $120 \mathrm{~h}$ para esta pesquisa.

A despesca foi realizada utilizando-se uma rede de nylon simples e um reservatório de plástico. Os indivíduos juvenis retidos na rede foram contados manualmente.

O acompanhamento da qualidade da água durante o experimento foi realizado através da análise de parâmetros que pudessem apresentar características tóxicas para o camarão 
ou ter interesse para a recirculação, conforme sugerido por Tilley et al. (2002), Jackson et al. (2003) e Islam et al. (2004); foram eles: amônia, nitrato, nitrito, fósforo, ortofosfato e clorofila a. As determinações foram feitas a partir de amostras compostas com água dos dois reservatórios da duplicata. As amostras foram refrigeradas e transportadas para o laboratório. As determinações foram realizadas no máximo $6 \mathrm{~h}$ após a coleta e seguiram os métodos recomendados em APHA (1995).

\section{Etapa 3 - Produção de camarão em um viveiro experimental}

Para avaliação da sobrevivência de pós-larvas e produtividade do camarão cultivado com águas de despesca, foi construído um viveiro experimental de $400 \mathrm{~m}^{2}$, com profundidade de aproximadamente $1 \mathrm{~m}$, o qual foi abastecido com a água proveniente do canal de descarga dos viveiros comerciais.

As pós-larvas foram aclimatadas no berçário da fazenda, durante 16 dias, antes do povoamento. Os dois viveiros (comercial e experimental) foram povoados com a mesma densidade populacional, 35 pós-larvas por metro quadrado. As pós-larvas receberam alimentação, aeração e tratos culturais iguais aos dos viveiros comerciais.

No viveiro experimental foram instaladas 4 gaiolas de $1 \mathrm{~m}^{2}$ para que, no primeiro mês após o povoamento, houvesse acompanhamento semanal da sobrevivência e peso médio das pós-larvas, em diferentes estágios. Após a despesca de cada gaiola os indivíduos vivos eram recolocados no tanque, para contagem na despesca final.

Após o primeiro mês foram realizadas visitas quinzenais à fazenda, para acompanhamento da sobrevivência e biometria dos camarões e avaliado o peso médio das pós-larvas de um dos viveiros comerciais e do viveiro experimental. Ao longo de todo o experimento foram realizadas cinco coletas de água de cada viveiro e do rio para análise dos parâmetros: $\mathrm{pH}, \mathrm{CE}, \mathrm{Ca}, \mathrm{Mg}, \mathrm{K}$, Na, NH3, Nitrato, RAS, soma de ânions e soma de cátions. Para a análise estatística dos dados o experimento foi considerado inteiramente casualizado com três tratamentos (três locais diferentes de coleta) e cinco repetições (número de coletas); para comparação de médias realizou-se o teste de Tukey.

\section{RESULTADOS E DISCUSSÃO}

\section{Etapa 1 - Teste de toxicidade in vitro}

A Tabela 3 apresenta o percentual de sobrevivência de cada tratamento. Nota-se que o maior percentual de sobrevivência no período estudado $(96,7 \%)$ ocorreu no tratamento com $100 \%$ de água de despesca. Os tratamentos com 75 e 50\% de água de despesca também apresentaram baixos índices de mortalidade; no entanto, não há uma correlação clara entre quantidade de água de despesca e mortalidade das pós-larvas ao longo de sete dias. Observa-se pequena diminuição na mortalidade quando se aumenta a proporção de água de despesca, porém esta diferença não é significativa ( $\mathrm{p}>0,05)$, indicando que a mortalidade apresentada é natural nessas condições ambientais e esta água pode ser reutilizada para o cultivo do camarão, em águas interiores. Os valores de $\mathrm{pH}$ foram mantidos em níveis ótimos, variando de 7,09 a 8,26.
Tabela 3. Sobrevivência de pós-larvas de camarões da espécie Litopenaeus vannamei durante sete dias

\begin{tabular}{llcc} 
& Tratamento & \multicolumn{2}{c}{ Sobrevivência } \\
\cline { 3 - 4 } & & $\begin{array}{c}\text { Número de } \\
\text { indivíduos (média) }\end{array}$ & $\%$ \\
T 1 & $100 \% A R$ & 18 & 88,3 \\
T 2 & $75 \% A R+25 \% A D$ & 18 & 91,7 \\
T 3 & $50 \% A R+50 \% A D$ & 19 & 95,0 \\
T 4 & $25 \% A R+75 \% A D$ & 19 & 93,3 \\
T 5 & $100 \% A D$ & 19 & 96,7 \\
\hline
\end{tabular}

Obs.: Número de indivíduos representa a média de sobrevivência no teste em triplicata. AR - Água do Rio Jaguaribe; AD - Água de despesca

\section{Etapa 2 - Teste de toxicidade in loco}

O índice de sobrevivência das pós-larvas e a descrição da nomenclatura utilizada para as amostras estão apresentados na Figura 2. A caracterização físico-química das amostras líquidas nos reservatórios, no início e no fim do experimento está apresentada na Tabela 4.

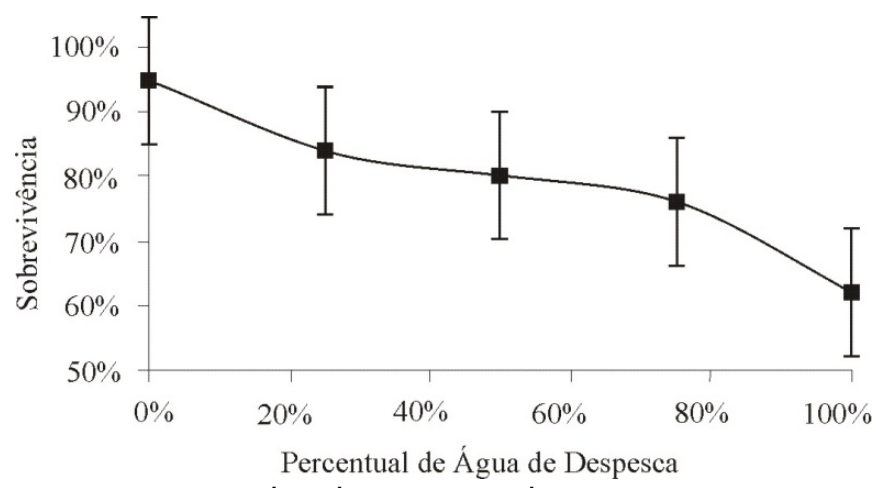

Figura 2. Taxa de sobrevivência dos camarões (juvenis) nos reservatórios, a partir das 50 unidades povoadas no início do experimento

Tabela 4. Caracterização das amostras dos reservatórios, no início e no final do experimento

\begin{tabular}{|c|c|c|c|c|c|c|}
\hline \multirow{2}{*}{ Amostra } & Amônia & $\mathrm{NO}_{3}^{-}$ & $\mathrm{NO}_{2}^{-}$ & $\mathbf{P}$ & $\mathrm{PO}_{4}^{-3}$ & \multirow{2}{*}{$\begin{array}{c}\text { Clor a } \\
\left(\mathrm{mg} \mathrm{m}^{3}\right)\end{array}$} \\
\hline & \multicolumn{5}{|c|}{$\left(\mathrm{mg} \mathrm{L}^{-1}\right)$} & \\
\hline \multicolumn{7}{|c|}{ Inicio do experimento } \\
\hline C & 0,1 & n.d. & 2,5 & n.d. & n.d. & 22,7 \\
\hline R1 & 0,4 & n.d. & 8,4 & n.d. & n.d. & 15,2 \\
\hline $\mathrm{R} 2$ & 0,6 & n.d. & 11,5 & n.d. & n.d. & 24,4 \\
\hline R3 & 0,1 & n.d. & 2,5 & n.d. & n.d. & 22,7 \\
\hline R4 & 0,1 & n.d. & 2,3 & n.d. & n.d. & 37,0 \\
\hline R5 & 0,1 & n.d. & 1,8 & n.d. & n.d. & 30,7 \\
\hline \multicolumn{7}{|c|}{ Final do experimento } \\
\hline C & 0,1 & n.d. & 2,5 & n.d. & n.d. & 22,7 \\
\hline $\mathrm{R} 1$ & 0,3 & n.d. & 2,2 & n.d. & n.d. & 82,8 \\
\hline $\mathrm{R} 2$ & 0,1 & n.d. & 2,9 & n.d. & n.d. & 22,4 \\
\hline R3 & 0,3 & n.d. & 1,9 & n.d. & n.d. & 27,7 \\
\hline R4 & 0,1 & n.d. & 3,2 & n.d. & n.d. & 25,6 \\
\hline R5 & 0,1 & n.d. & 2,9 & n.d. & n.d. & 4,0 \\
\hline
\end{tabular}

Um dos principais problemas na produção de camarão é a toxicidade dos resíduos nitrogenados presentes na água de cultivo, tais como amônia e nitrito, que podem levar à redução do crescimento e da sobrevivência (Montoya et al., 2002; Barajas et al., 2006). Amônia é o principal produto final do catabolismo protéico excretado por crustáceos e é altamente 
solúvel em água (Dall et al., 1990). Este composto pode provir também da amonificação de ração não consumida, resíduos orgânicos e sedimentos (Zhao et al., 1997; Barajas et al., 2006), podendo estar presente na forma ionizada $\left(\mathrm{NH}_{4}^{+}\right)$ou não ionizada $\left(\mathrm{NH}_{3}-\mathrm{N}\right)$. Se a concentração de $\mathrm{NH}_{3}-\mathrm{N}$ aumenta na água sua excreção pelo camarão diminui; em consequência, os níveis dessa substância aumentam no sangue e tecidos, com sérios efeitos na fisiologia (diminuição do crescimento), influenciando a mortalidade dos indivíduos (Neil et al., 2005; Colt \& Armstrong, 1981). Desta forma, a interação entre amônia e produção de camarão deve ser sempre considerada pelos carcinicultores, a fim de se obter o retorno financeiro esperado (Frias -Espericureta et al., 2000).

Diferente do que foi encontrado nos experimentos em escala de laboratório, quanto maior a proporção de água de despesca maior também foi a mortandade observada (maior potencial tóxico para a espécie). A partir de pesquisas com testes de toxicidade, Zhao et al. (1997) detectaram o nível seguro de amônia em 3,16 mg L-1 para indivíduos juvenis. Outros autores confirmam este valor como oscilando entre 3 e $4 \mathrm{mg} \mathrm{L}^{-1}$ (Maia et al., 2011). Contudo, comparando a Figura 2 com a Tabela 4 , infere-se que isto não ocorreu devido à amônia ou ao nitrito, que ficaram aquém dos limites considerados tóxicos.

Para Camargo et al. (2005), não se tem dado muita atenção à toxicidade do nitrato em animais aquáticos mas se tem dado aos efeitos tóxicos da amônia e do nitrito. Alguns autores chegam a considerar o nitrato como não tóxico ao camarão, ou prejudicial apenas em valores elevados, $50 \mathrm{mg} \cdot \mathrm{L}^{-1}$ (Boyd \& Clay, 2002). O aumento dos níveis de nitrato ocorre em sistemas de aquicultura em virtude da nitrificação e da adição de culturas de microalgas para alimentação (Muir et al., 1991). A principal ação tóxica do nitrato em animais aquáticos é a conversão dos pigmentos transportadores de oxigênio (hemoglobina e hemocianina) em formas incapazes de realizar esse transporte (metahemoglobina, por exemplo). O nível máximo aceitável de tolerância de animais aquáticos para o nitrato é de $2 \mathrm{mg} \mathrm{NO}_{3}^{-}$ $\mathrm{N} \cdot \mathrm{L}^{-1}$ (Camargo et al., 2005). Encontram-se, na literatura, limites máximos de $1 \mathrm{mg} \mathrm{NO}-\mathrm{N} \cdot \mathrm{L}^{-1}$ (Muir et al., 1991); entretanto, o nitrato não foi detectado neste estudo o que se levou a desconsiderá-lo parâmetro de toxicidade ou mortandade.

$\mathrm{O}$ fósforo não aparece na literatura em limites tóxicos para o camarão. Ao contrário, ele é necessário na formulação do alimento para o camarão (Montoya et al., 2000). entretanto, o excesso de fósforo no efluente constitui o principal fator de proliferação de algas e de eutrofização de ambientes aquáticos. Nesta pesquisa não foram detectados fósforo nem ortofosfato, visto que esses nutrientes não foram considerados fator limitante ao crescimento já que foram fornecidos às pós-larvas na ração diária.

Descartados os compostos nitrogenados ou fosforados como elementos de mortandade neste experimento, as algas surgem como principais e mais óbvios elementos tóxicos presentes nas águas de despesca porém a clorofila $a$ indicou a presença uniforme de algas verdes ao longo do experimento, em todos os reservatórios, as quais também foram excluídas como fator de toxicidade.

Por fim, as amostras foram levadas para análise microscópica cujos resultados mostraram que, quanto maior a porcentagem de água de despesca nos reservatórios maior também a quantidade de cianobactérias (algas verdes-azuis) presentes. Essas algas podem liberar cianotoxinas no meio, com efeito letal aos organismos, ou acumular neurotoxinas e hepatoxinas na cadeia trófica. De acordo com Alves \& Mello (2007), o cultivo de camarões em água de baixa salinidade favorece a floração dessas algas

\section{Etapa 3 - Produção de camarão em um viveiro experimental}

As pós-larvas que estavam ambientadas no viveiro experimental, apresentaram peso médio superior ao das que estavam ambientadas no viveiro comercial com água do rio (Figura 3). Os camarões cultivados no efluente alcançaram o peso médio de corte de $8 \mathrm{~g}$ em 68 dias (Tabela 5) enquanto os camarões cultivados em água do rio só atingiriam este peso aos 116 dias. Esses resultados demonstram que os nutrientes presentes no efluente podem contribuir para a engorda do camarão da espécie Litopenaeus vannamei cultivado em águas interiores e que o reúso desta água pode ser uma alternativa viável, já que aumenta a produtividade de camarão através da diminuição do tempo de engorda em até um mês e meio o que permite, ao produtor, aumentar o número de ciclos por ano de 3 para 4.

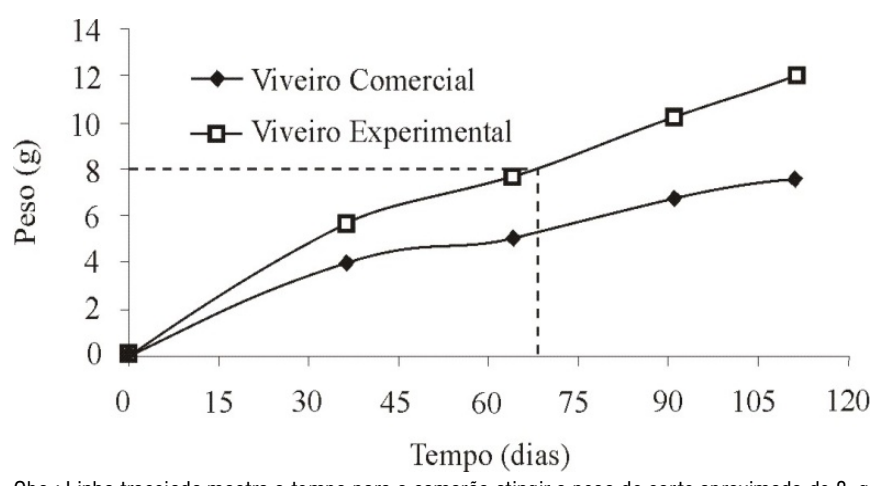

Obs.: Linha tracejada mostra o tempo para o camarão atingir o peso de corte aproximado de $8 \mathrm{~g}$

Figura 3. Crescimento do camarão dos viveiros comercial e experimental

Tabela 5. Resultados comparativos de povoamento e de despesca em viveiros abastecidos com efluente da carcinicultura e água do Rio Jaguaribe

\begin{tabular}{lcc}
\hline \multirow{2}{*}{ Dados } & \multicolumn{2}{c}{ Água utilizada no viveiro } \\
\cline { 2 - 3 } & AD & AR \\
Povoamento & & \\
Quantidade Total de Pós-larvas & 14.100 & 1.050 .000 \\
Quantidade de Pós-larvas por m ${ }^{2}$ & 35 & 35 \\
Peso Total $(\mathrm{g})$ & 705 & 52.500 \\
\hline Despesca & & \\
Produção $(\mathrm{kg})$ & 71,0 & $5.362,5$ \\
Peso médio $(\mathrm{g})$ & 11,9 & 7,7 \\
Quantidade de camarões & 5.972 & 696.429 \\
Tempo para atingir 8 g (dias) & 68 & 116 \\
Percentual de sobrevivência & 42,1 & 66,3 \\
\hline
\end{tabular}

Obs.: $A D=$ Água de despesca; $A R=$ Água do rio Jaguaribe

Na Tabela 5 estão os resultados comparativos entre o povoamento e a despesca nos dois viveiros (comercial e experimental). $\mathrm{O}$ viveiro comercial teve taxa de sobrevivência 
de $66,32 \%$ e o viveiro experimental, $42,1 \%$. Segundo o proprietário da Fazenda Poço da Onça, este índice pode ser considerado bom já que é possível diminuir os custos com água e energia para bombeamento em até $50 \%$, além de ajudar a diminuir a carga poluidora lançada no rio Jaguaribe, já que cada despesca pode lançar cerca de $30.000 \mathrm{~m}^{3}$ de água com altas concentrações de nitrogênio de fósforo (Tabela 4), aumentando o risco de eutrofização dos corpos d'água.

De acordo com Fonseca et al. (2009), os aumentos semanais do peso dos camarões variam em função das densidades de estocagem, período de mudas, eficiência da conversão alimentar, qualidade das rações, espécie e qualidade das águas. Os valores médios de engorda dos camarões em fazendas variam entre 0,70 a 1,20 g por semana. As taxas de mortalidade semanal oscilam entre 1 a $3 \%$, considerando as perdas provocadas por predadores naturais e a ação do canibalismo. Todavia, ao se atingir o tamanho comercial entre 11 e 12,5 g, a sobrevivência final média em cultivos semi-intensivos é de $65 \%$ e a média de crescimento semanal de $0,8 \mathrm{~g}$.

Na Tabela 6 são apresentados os valores médios de algumas características químicas da água do Rio Jaguaribe, do viveiro comercial e do viveiro experimental observados durante o experimento. Não foram constatadas diferenças estatísticas entre as águas com relação aos níveis de $\mathrm{pH}$, soma de ânions, soma de cátions, $\mathrm{Ca}^{2+}, \mathrm{Mg}^{2+}, \mathrm{K}^{+}, \mathrm{Na}^{+}, \mathrm{N}$-amoniacal, $\mathrm{N}$-nitrato, condutividade elétrica ( $\mathrm{CE}_{\mathrm{a}}$ ) e Razão de Adsorção de Sódio (RAS).

Tabela 6. Médias ${ }^{1}$ das características químicas da água do rio, do viveiro comercial e do viveiro experimental

\begin{tabular}{lccc}
\hline \multicolumn{1}{c}{ Parâmetros } & Rio & $\begin{array}{c}\text { Viveiro } \\
\text { comercial }\end{array}$ & $\begin{array}{c}\text { Viveiro } \\
\text { experimental }\end{array}$ \\
$\mathrm{pH}$ & 7,47 & 7,57 & 7,48 \\
Soma ânions $\left(\mathrm{mmol}_{\mathrm{c}} \mathrm{L}^{-1}\right)$ & 5,95 & 5,62 & 6,15 \\
Soma cátions $\left(\mathrm{mmol}_{\mathrm{c}} \mathrm{L}^{-1}\right)$ & 5,53 & 4,87 & 5,43 \\
$\mathrm{Ca}^{2+}\left(\mathrm{mmol}_{\mathrm{c}} \mathrm{L}^{-1}\right)$ & 1,21 & 1,07 & 1,11 \\
$\mathrm{Mg}^{2+}\left(\mathrm{mmol}_{\mathrm{c}} \mathrm{L}^{-1}\right)$ & 0,89 & 0,81 & 0,86 \\
$\mathrm{~K}^{+}\left(\mathrm{mmol}_{\mathrm{c}} \mathrm{L}^{-1}\right)$ & 0,18 & 0,18 & 0,20 \\
$\mathrm{Na}^{+}\left(\mathrm{mmol}_{\mathrm{c}} \mathrm{L}^{-1}\right)$ & 3,25 & 2,80 & 3,26 \\
$\mathrm{~N} \mathrm{Amoniacal}\left(\mathrm{mg} \mathrm{L}^{-1}\right)$ & 0,86 & 1,38 & 1,34 \\
$\mathrm{~N} \mathrm{Nitrato}\left(\mathrm{mg} \mathrm{L}^{-1}\right)$ & 1,14 & 0,88 & 1,32 \\
$\mathrm{CE}$ & $\left.0,5 \mathrm{dS} \mathrm{m})^{-1}\right)$ & 0,55 & 0,60 \\
$\mathrm{RAS}\left(\mathrm{mmol} \mathrm{L}^{-1}\right)^{0,5}$ & 3,26 & 2,86 & 3,26 \\
\hline
\end{tabular}

${ }^{1}$ Médias das linhas são semelhantes, segundo 0 teste de Tukey $(P>0,05)$

O acompanhamento da taxa de mortalidade do viveiro experimental foi realizado através das gaiolas instaladas dentro do viveiro, em quatro pontos distintos. Na gaiola I, que foi "despescada" uma semana após o povoamento, a mortalidade foi de $45,71 \%$; na semana seguinte a mortalidade aumentou $5,72 \%$, na terceira semana aumentou $2,81 \%$ e na quarta mais $2,80 \%$. Assim, a maior taxa de mortalidade apresentada pelas pós-larvas cultivadas no efluente ocorreu na primeira semana após o povoamento devido, provavelmente, à alta sensibilidade das pós-larvas (ou indivíduos juvenis) às substâncias tóxicas presentes na água reutilizada, o que pode ser um indicativo de mudança de estratégia de produção de camarão. Talvez seja possível povoar os viveiros com densidade maior, prevendo que haverá alta mortalidade na primeira semana de cultivo; desta forma, poder-se-á compensar a perda de indivíduos e ainda obter a vantagem de diminuição no tempo do ciclo de engorda.

\section{CONCLusões}

1. No teste de toxidade in vitro não foi observada diferença significativa entre as taxas de sobrevivência das pós-larvas nas diferentes concentrações de água.

2. No teste de toxicidade in loco acredita-se que as cianobactérias foram responsáveis pela toxicidade constatada no experimento, possivelmente através da liberação de cianotoxinas no meio. Contudo, é necessário um estudo mais aprofundado para estabelecer a relação entre a concentração das cianotoxinas e a mortandade de espécies.

3. É possível o reúso de água de despesca da carcinicultura para o cultivo de camarão, com índice de sobrevivência de $42,1 \%$.

4. O teste usando um viveiro experimental mostrou que o uso da água de despesca acelerou o crescimento dos camarões, que alcançaram o peso médio de corte de $8 \mathrm{~g}$ em 68 dias, enquanto usando água do rio este peso somente foi atingido em 116 dias;

5. A mortalidade dos camarões ocorre de início, na primeira semana do ciclo de engorda, indicando que uma mudança de estratégia na produção de camarão pode aumentar a produtividade da fazenda.

\section{LITERATURA CITADA}

Alencar, J. R.; Horta Júnior, P. A.; Celino, J. J. Cultivo de camarão branco Litopenaeus Vannamei (Boone, 1931) com a Macroalga Ulva Lacuata Linneaus (Chlorophyta) no tratamento de efluentes em sistema fechado de recirculação. Revista de Biologia e Ciências da Terra, v.10, p.117-137, 2010.

Allan, G. L.; Maguire, G. B.;Hopkins, S. J. Acute and chronic toxicity of ammonia to juvenile Metapenaeus macleayi and Penaeus monodon and the influence of low dissolvedoxygen levels. Aquaculture, v.91, p.265-280, 1990.

Alves, C. S.; Mello G. L. Manual para o monitoramento hidrobiológico em fazendas de cultivo de camarão. Recife: FAEPE/SEBRAE-PE. 2007. 58p.

APHA - American Public Health Association. Standard methods for the examination of water and wastewater. Washington:APHA, 25.ed., 1995. 1298p.

Barajas, F. J. M.; Villegas, R. S.; Clark, G. P.; Moreno, B. L. Litopenaeus vannamei (Boone) post-larval survival related to age, temperature, $\mathrm{pH}$ and ammonium concentration. Aquaculture Research, v.37, p.492-499, 2006.

Boyd, C. E.; Clay, J. W. Evaluation of belize aquaculture, Ltd: A superintensive shrimp aquaculture system. Report prepared under the World Bank, NACA, WWF and FAO Consortium. Program on shrimp farming and the environment. Washington: FAO, 2002.17p. 
Brasil. Resolução CONAMA n. 312 de 10 de outubro de 2002. Dispõe sobre parâmetros, definições e limites de áreas de preservação permanente. Diário Oficial da União, n.224, s.1, p.92-95, 2002.

Camargo, J. A.; Alonso, A.; Salamanca, A. Nitrate toxicity to aquatic animals: A review with new data for freshwater invertebrates. Chemosphere, v.58, p.1255-1267, 2005.

Castellani, D.; Camargo, A. F. M.; Abimorad, E. G. Aquaponia: Aproveitamento do efluente do berçário secundário do Camarão-da-Amazônia (Macrobrachium amazonicum) para produção de alface (Lactuca sativa) e agrião (Rorippa nasturtium aquaticum) hidropônicos. Bioikos, v.23, p.6775, 2009.

Castello, J. P.; Poersch, L.; Vasconcellos, M. C.; Cavalli, R.; Wasielesky, W. Rearing shrimps in pens: A predictive model for impact assessment. Estuaries and Coasts, v.31, p.215-222, 2008.

Colt, J.; Armstrong, D. A. Nitrogen toxicity to crustaceans, fish, and molluscs. In: Allen, L. J.; Kinney, E. C. Proceeding of the Bio-Engineering Symposium for Fish Culture, American Fisheries Society, Fish Culture Society, FCS Publ. 1, 1981, p.34-47.

Dall, W.; Hill, B. J.; Rothlisberg, P. C.; Staples, D. J. The biology of the Penaeidae. In: Blaxter, J. H. S.; Southward, A. J. (ed.) Advances in marine biology. San Diego: Academic Press, v.27, 489p. 1990.

Figueiredo, M. C. B.; Araújo, LF. P.; Rosa, M. F.; Morais, L. F. S.; Paulino, W. D.; Gomes, R. B. Impactos ambientais do lançamento de efluentes da carcinicultura de águas interiores. Revista Engenharia Sanitária e Ambiental, v.11, p.231-240, 2006.

Figueiredo, M. C. B., Rosa, M. F.; Gondim, R. S. Gestão da demanda hídrica em municípios do médio e baixo Jaguaribe. In: Encontro Nacional sobre gestão empresarial e meio ambiente, São Paulo. Anais.... FGV/FAPESP/USPFEA/USP. 2003. CD-Rom

Fonseca, S. B.; Mendes, P. P.; Albertim, C. J. L.; Bittencourt, C. F; Silva, J. H. V. Cultivo do camarão marinho em água doce em diferentes densidades de estocagem. Pesquisa Agropecuária Brasileira, v.44, p.1352-1358, 2009.

Frias-espericureta, M. G.; Harfush-melendez, M.; Páez-osuna, F. Effects of ammonia on mortality and feeding of postlarvae shrimp Litopenaeus vannamei. Bulletin of Environmental Contamination and Toxicology, v.65, p.98$103,2000$.

Islam M. S.; Khan, S.; Tanaka, M. Waste loading in shrimp and fish processing effluents: Potential source of hazards to the coastal and nearshore environments. Marine Pollution Bulletin, v.49, p.103-110, 2004.

Jacson, C. J.; Preston, N.; Buford, M. A.; Thompson, P. J. Managing the development of sustainable shrimp farming in Australia: The role of sedimentation ponds in treatment of farm discharge water. Aquaculture, v.226, p.23-34, 2003.

Joventino, F. K. P.; Mayorga, M. I. O. Diagnóstico socioambiental e tecnológico da carcinicultura no Município de Fortim, Ceará, Brasil. Revista Eletrônica do Prodema, v.2, p.86-96, 2008.

Lin, Y. F.; Jing, S. R.; Lee, D. Y. The potential use of constructed wetlands in a recirculating aquaculture system for shrimp culture. Environmental Pollution, v.123, p.107-113, 2003.
Maia, E. P.; Gàlvez, A. O.; Silva, L. O. B. Brazilian shrimp farms for Litopenaeus vannamei with partial and total recirculation systems. International Journal of Aquatic Science, v.2, p.1626, 2011.

Miranda, F. R.; Cavalvante, R. R. R.; Ribeiro, E. M.; Lima, R. N. Uso de efluentes da carcinicultura na irrigação de Panicum maximum cvs. Tanzânia e Mombaça. Revista Ciência Agronômica, v.41, p.46-52, 2010.

Miranda, F. R.; Tavares, R. C.; Lima, R. N.; Crisóstomo, L. A. Uso de efluente da carcinicultura de águas interiores na irrigação de arroz e melão. Fortaleza: Boletim de Pesquisa e Desenvolvimento da Embrapa Agroindústria Tropical, n.28, 2007.23p.

Montoya, R. A.; Lawrence, A. L.; Grant, W. E.; Velasco, M. Simulation of phosphorus dynamics in an intensive shrimp culture system: effects of feed formulations and feeding strategies. Ecological Modelling, v.129, p.131-142, 2000.

Montoya, R. A.; Lawrence, A. L.; Grant, W. E.; Velasco, M. Simulation of inorganic nitrogen dynamics and shrimp survival in an intensive shrimp culture system. Aquaculture Research v.33, p.81-94, 2002.

Muir, P. R.; Sutton, D. C.; Owens, L. Nitrate toxicity to Penaeus monodon Protozoea. Marine Biology, v.108, p.67-71, 1991.

Neil, L. L.; Fotedar, R.; Shelley, C. C. Effects of acute and chronic toxicity of unionized ammonia on mud crab, Scylla serrata (Forsskål, 1755) larvae. Aquaculture Research, v.36, p.927932, 2005.

Sandu, S. I.; Boardmanb, G. D.; Wattenc, B. J.; Brazil, B. L. Factors influencing the nitrification efficiency of fluidized bed filters with a plastic beans medium. Aquacultural Engineering, v.26, p.41-59, 2002.

Soares, A. M. L.; Carvalho, M. S. B. de S.; Barreto, R. N. da C.; Soares, Z. M. L. Análise temporal do crescimento da carcinicultura marinha no estuário do rio Jaguaribe-Ceará. In: Simpósio Brasileiro de Sensoriamento Remoto, 13, 2007. Florianopolis. Anais....Florianópolis: INPE, 2007. p.4267-4274.

Spanghero, D. B. N.; Silva, U. L.; Pessoa, M. N. C.; Medeiros, E. C. A.; Oliveira, I. R.; Mendes, P. P. Utilização de modelos estatísticos para avaliar dados de produção do camarão Litopenaeus vannamei cultivados em águas oligohalina e salgada. Acta Scientiarum, v.30, p.451-458, 2008.

Tilley, D. R.; Badrinarayanan, H.; Rosati, R.; Son, J. Constructed wetlands as recirculation filters in large-scale shrimp aquaculture. Aquacultural Engineering, v.26, p.81-109, 2002.

Valenti, W. C. Criação de camarões de água doce. In: Congresso de Zootecnia, 120, Vila Real, Portugal, 2002, Vila Real: Associação Portuguesa dos Engenheiros Zootécnicos. Anais... 2002, p. 229-237.

Wajsbrot, N.; Gasith, A.; Krom, M. D.; Samocha, T. M. Effect of dissolved oxygen and the molt stage on the acute toxicity of ammonia to juvenile green tiger prawn Penaeus semisulcatus. Environmental Toxicology and Chemistry, v.9, p.497-504, 1990.

Wang, J. K. Conceptual design of a microalgae-based recirculating oyster and shrimp system. Aquacultural Engineering, v.28, p.37-46, 2003.

Zhao, J. H.; Lam, T. J.; Guo, J. Y. Acute toxicity of ammonia to the early stage-larvae and juveniles of Eriocheir sinensis H. Milne-Edwards, 1853 (Decapoda: Grapisidae) reared in the laboratory. Aquaculture Research, v.28, p.517-525, 1997. 